\title{
Editorial
}

\section{Comentario editorial sobre "Tratamiento laparoscópico de la ureterolitiasis: nuestra experiencia"}

\author{
En referencia al artículo publicado en este número de Actas en las páginas 667-669
}

\author{
Álvaro Serrano Pascual
}

Servicio de Urología. Hospital Universitario de Guadalajara. Guadalajara, España.

$\mathrm{E}^{1}$ tratamiento de la litiasis ureteral ha variado a lo largo del tiempo. Si bien, el tratamiento quirúrgico mediante cirugía abierta, era la elección antes de la década de los 80 , en ésta se producen dos acontecimientos que variaran el curso de la historia en la terapéutica de la litiasis ureteral: el descubrimiento y desarrollo del ureteroscopio rígido por E. Pérez-Castro ${ }^{1}$ y el descubrimiento y aplicación de las ondas de choque para el tratamiento de la litiasis, por Chaussy ${ }^{2}$. Desde entonces, se han realizado múltiples estudios para determinar la eficacia de cada una de las técnicas en el tratamiento de la litiasis ureteral, teniendo en cuenta diversos parámetros, entre los que destacan la tasa libre de cálculos, el número de procedimientos necesarios, los costes y, por último, el número de complicaciones. Por otra parte, con el nacimiento y desarrollo de la laparoscopia se han incluido nuevas indicaciones terapéuticas, entre las que se encuentran las litiasis ureterales.

En las Guías Clinicas sobre Urolitiasis más recientes $^{3,4}$ se establece una serie de niveles de evidencia y recomendaciones en cuando al tratamiento de la litiasis ureteral, e indican que tanto la Ureteroscopia (URS) como la Litotricia Extracorpórea por Ondas de Choque (LEOC) son aceptables como tratamiento de primera línea (Nivel de Evidencia 1A), aunque con la URS se obtiene una tasa libre de cálculos mayor que con la LEOC, en todas las partes del uréter, excepto en el uréter proximal asociado a cálculos mayores de 10 milímetros. Sin embargo, estas Guías Clínicas consideran que la Ureterolitomía Laparoscópica (UL) puede ser una opción en casos de litiasis ureterales grandes e impactadas con fracaso de la LEOC, la URS retrógrada y la URS anterógrada, y siempre antes que recurrir a la cirugía abierta.

En el presente trabajo, los autores nos exponen los resultados del tratamiento efectuado a un número importante de casos de litiasis ureterales del ter- cio medio y superior, mediante UL, en relación a otras serie publicadas ${ }^{5-8}$. El tamaño de los cálculos es grande, media de 13 milímetros, pero observamos, aunque no existe detalle del tamaño de los cálculos tratados, que hay algunos menores de 10 milímetros y, se considera que éstos serían subsidiarios de tratamiento con URS o LEOC. Asimismo, se observa en el trabajo que la UL ha sido indicada, como tratamiento de primera línea, en un alto porcentaje de pacientes (54,5\% de los casos). Aunque la UL es una técnica altamente eficaz, no es un tratamiento de primera línea en la mayoría de los casos de los cálculos ureterales, por su mayor invasividad, más largo tiempo de recuperación postoperatoria y mayor número de complicaciones asociadas que con la URS o la LEOC. Por otra parte, según refieren los autores, en los países en desarrollo los costes de la URS o la LEOC pueden ser superiores a la UL, lo cual puede ser una justificación, pero considero que ello no debe ser del todo determinante.

Con respecto a la vía elegida para realizar la UL es de destacar que los autores utilizan de forma preferente la vía transperitoneal. En mi opinión la vía retroperitoneal, aunque más difícil técnicamente, puede ser más beneficiosa para el paciente, sobre todo en aquellos casos que pueda haber orina infectada y exista un extravasado de la misma; sin embargo, hay que tener en cuenta que una sutura laparoscópica cuidadosa puede evitar fístulas urinarias, y que en caso de dudas de la estanqueidad de la sutura o que el uréter se encuentre en malas condiciones, el dejar colocado un catéter doble J puede evitar extravasados urinarios.

Con los recientes desarrollos de los ureteroscopios, sobre todo, en disminución del calibre y, la utilización del láser Holmium para la fragmentación de los cálculos ureterales ${ }^{9}$, los algoritmos han cambiado, por ser la URS más eficiente, tener menor coste y ser una cirugía mínimamente invasiva; no obstan- 
te, considero que las decisiones del tratamiento de la litiasis ureteral deben ser tomadas de forma individualizada y basadas en la preferencia de los pacientes, la experiencia personal y el equipamiento local, y esto es justamente lo que en el presente trabajo se expone con unos resultados buenos $(90,9 \%)$ utilizando laparoscopia para el tratamiento de cálculos ureterales grandes e impactados, los cuales pueden plantear problemas clínicos importantes.

Finalmente, coincido con Borges et al. que las indicaciones de la ureterolitomía laparoscópica son las mismas que las de la cirugía abierta, pero además la UL aporta las ventajas de la cirugía mínimamente invasiva de menor requerimiento analgésico, tener menor estancia hospitalaria y disminuir el tiempo hasta recobrar la actividad normal.

\section{REFERENCIAS}

1. Pérez-Castro E, Martinez-Piñeiro JA. Ureteral and renal ureteroscopy: a new approach. Eur Urol. 1982;8(2):117-120.

2. Chaussy C, Schmiedt E, Jocham D, Brendel W, Forssmann B, Walter V. First clinical experience with extracorporeally induced destruction of kidney stones by shock waves. J Urol. 1982;127 (3):417-420.

3. Preminger GM, Tiselius HG, Assimos DG, Alken P, Buck C, Gallucci M, et al. 2007 Guideline for the management of ureteral calculi. J Urol. 2007;178(6):2418-2434.
4. Tiselius HG, Ackermann D, Alken P, Buck C, Conort P, Gallucci M, et al. Guidelines on urolithiasis. Eur Urol. 2001;40(4):362371.

5. Fariña Pérez LA, Pesqueira Santiago D, Meijide Rico F, Zungri Telo ER. Ureterolitotomia laparoscópica de un cálculo de uréter iliaco olvidado durante más de ocho años. Actas Urol Esp. 2006;30(2):218-21.

6. El-Moula MG, Abdallah A, El-Anany F, Abdelsalam Y, Abolyosr A, Abdelhameed D, et al. Laparoscopic ureterolithotomy: Our experience with 74 cases. Int J Urol. 2008;15(7):593-597.

7. Derouiche A, Belhaj K, Garbouj N, Hentati H, Ben Slama MR, Chebil M. [Retroperitoneal laparoscopy for the management of lumbar ureter stones. Prog Urol. 2008;18(5):281-287.

8. Basiri A, Simforoosh N, Ziaee A, Shayaninasab H, Moghaddam SM, Zare S. Retrograde, antegrade, and laparoscopic approaches for the management of large, proximal ureteral stones: a randomized clinical trial. J Endourol. 2008;22(12):2677-2680.

9. Soler M, Watterson JD, Wollin TA, Mott L, Razvi H, Denstedt JD. Holmium: YAG laser lithotripsy for upper urinary tract calculi in 598 patients. J Urol. 2002;167(1):31-34.

Correspondencia autor: Dr. Álvaro Serrano Pascual Servicio de Urología

Hospital Universitario de Guadalajara Donantes de Sangre, s/n. 19002 Guadalajara.

Tel.: 949209200

E-mail autor: alvaro.serrano.p@gmail.com

Información artículo: Editorial

Trabajo recibido: marzo 2009

Trabajo aceptado: abril 2009 